\title{
The Semiotic Study of Rag-E Khab from the Peirce's Perspective
}

\author{
Dr. Foroogh kazemi ${ }^{1 *}$, Shohreh Dalaee ${ }^{2}$ \\ ${ }^{1}$ Associate professor of linguistics, Central Tehran Branch, Islamic Azad University Tehran, Iran \\ ${ }^{2}$ M.A of Linguistics, Central Tehran Branch, Islamic Azad University, Tehran, Iran
}

*Corresponding Author: Dr. Foroogh kazemi, Associate professor of linguistics, Central Tehran Branch, Islamic Azad University Tehran, Iran

\begin{abstract}
The purpose of this study was to investigate the semiotic aspects of the movie Rag-e Khab. In this research, the film sequences formed the research data, the research method was descriptive-analytic and the data were evaluated based on the Peirce's semiotic view. The present research pursues to answer the questions like which signs are used and how is the representation of gender in these sequences? The findings of the research indicate that in these sequences, in addition to the icon signs, index signs are used more and the symbolic signs are used lesser. The results also revealed that the representation of gender in this film is female-centered and portrayed women's lives and their problems.
\end{abstract}

Keywords: Peirce's semiotic view, Types of signs, Rag-e Khab movie.

\section{INTRODUCTION}

Semiotics is a science that studies the structures of signs. This branch of science has been used since the 1950s as a research method in recognizing the signification and perceptions of communication functions. Philosophers such as Michael Foko, Ferdinand Saussure, Charles Sanders Peirce, and Roland Barth have made significant contributions to this field, and in the particular context of a semiotics of the image, which is a branch of the science of identifying visual semiotics, important works are written by Barth on photography, cinema and painting.

The purpose of semiotics is to study sign structures such as languages, ciphers, symbols, marked signs, and all kind of this effects. Semiotics is one of the best and most effective methods, which involves with meaning-making or in the words of Roland Barth, "process of being meaningful". Codes are widespread and socialized systems of conventions in the field of signs, where each layer of the text is received through conventions of a distinct code. Also, in a text, we encounter the interaction between different meanings and multilateral interaction between different codes, which results in multiplicity of semantics (Sojoodi, 2008: 230).

Peirce, introduced a theory about signs, which he also called semeiotics. His view is based on the logical function of the signs. The field of signification is important to him, to the extent that, he considers semiotics as the knowledge of the investigating all signifier systems. Nowadays, movie semiotics, such as an approach to text analysis, can bring us closer to the meaning spectrum of the movie. Sign is a symbol that refers to the expression of a term or the induction of a concept, and semiotics is a science that examines the different types of signs, the factors present in the process of their production and their interpretation, as well as the rules governing them. In other words, the semiotics science is the sign cognition science, and its purpose is to identify the orders and rules that bring meanings together. The present research seeks to answer to these questions: What kind of signs can be represented in this movie in accordance with Peirce's approach? How many of each sign have been used? And regarding the types of signs in this movie how is gender representation? Life of today's human is surrounded by numerous visual signs that reading them is inevitable. In these times that movies speak to us like writing sand infiltrate every fiber of our lives, recognizing the relationship of signs in the movie and its social functions can enlighten many of the implications of the social and historical phenomena of society, therefore, it is important to address such a research. In this research, library method is used to collect data. The research corpus consists of sequences from the movie Rag-e Khab directed by Hamid Nematollah, which are collected and analyzed. 


\section{REVIEW OF RELEVANT STUdIES}

Every artwork is a sign pointing to the world of ideas. Of Locke-Peirce-Morris (1963) intellectual tradition territories, non-verbal human communications like gestures and facial expressions (gesturalpostural study), as well as spatial interaction techniques (spatial study) can be mentioned and this is the territory in which semioticians encounter with the thoughts of human behavior anthropologists. In an adaptive study, Kazemi and Ghasemi Ateni (2016) investigated various types of signs in photos of Reuter's news from Peirce's and Bergen's points of view. Findings of this study showed that in the photographs of this news agency, out of 30 photos, Bergen's and Peirce's views were similar in 14 photos, and in the 16 photos their views contradicted each other.

Sebeok (2001) has explained basic notions of semiotics. He has introduced the object of semiotics and has written about the semiosis and representation, types of signs and nonverbal communication. In his book, Sebeok has explained about a biological approach to the study of signs and has mentioned about sings and reality. Furthermore, he has introduced symptom signs, Fetish signs, language signs and language as a primary modeling system.

Sharam and Gupta (2015) mentioned that large part of any individual is surrounded by lot of signs and symbols, however, the way they comprehend these sign, symbols and meaning differ from one to other individual. Since, India is a vast country with vivid and varied culture and demographics, it becomes a challenge for advertisers to target and attract right consumers through their advertisements. In such a situation it is essential for advertisers to understand he choice consumers have for advertisements and the differential impact it has on the consumers. Their study shows the differential impact advertisements have through their themes, colors, to be more specific, impact that semiotics have on consumers and how it can be made more effective and targeted by understanding the language and impact of semiotics on consumers in India.

\section{THEORETICAL FRAMEWORK}

Semiotics has a long history and is not a theory that has been formed at once. But methodologists go over the traces of the theory in Ferdinand Saussure's and Charles Sanders Peirce's approaches. The first has more technical and linguistic perspectives on this theory, and the latter has a more flexible approach. In fact, both of them have established a new link between knowledge and sign, and have introduced this intellectual approach to the domain of knowledge studies (Zamiran, 2003: 8).

Peirce, unlike Saussure, does not refer to sign itself, but rather to the process of producing and interpreting signs or so called, semiosis, placing it at the center of his semiotic theory. Semiosis requires three elements of sign, object, and interpretation. The semiosis process associates the signs with examples. In this process, the sign is "something that, in a particular direction and as a subject, sits in place of something in someone's opinion." According to Saussure, sign has a dual nature, on one side of which is a signifier or a phonetic image of the word, and on the other hand, is a signified or a mental and conceptual image of it. Neither of these is singly the sign, but their interconnected and correlated structural relation, which is called signification, creates the sign, a relationship that is essentially arbitrary. Saussure considered the sign as a combination of signifier and signified. He considered signifier as a phonetic image and signified as a mental image. Peirce's semiotic theory is based on the interaction and the trichotomy relations of the elements, and it cannot be reduced to the Saussure's dual relation. Peirce introduced a three-sided model:

- Representament (appearance): The form which the sign takes (and is not necessarily material).

- Interpretation (explanation): Not interpreter, but the meaning that is obtained from the sign.

- The object (object): that which the sign represents.

Let's look at the types of these signs from the Peirce's (1931) point of view:

\subsection{Iconic Sign}

It is based on the similarity of the relation between the signifier and signified or sign and the subject. Pictures, caricatures, models and onomatopoeias, metaphors, and the use of internal sounds in music are examples of this type. 


\subsection{Index Sign}

It is based on a kind of causal or physical relationship between the signifier and the signified. These relations are not arbitrary, such as smoke that is a sign of fire or personal pronouns, and time and space constraints in language.

\subsection{Symbolic Sign}

It is a kind of conventional or arbitrary relationship, which depends on the signified. Among these language signs, musical notes, and Morse's alphabet can be mentioned. Let us remember that implicit significations play an important role in symbolic signs.

\section{Data Analysis}

In this section, we present some examples of analyzed sequences of the movie, and then we will summarize the results of analysis of the entire data in a table and graph, but before discussion about results of analysis, a summary is brought of the content of the film as follows:

\subsection{Rag-e Khab}

Mina has abandoned her undergraduate studies and is looking for a job after separation from her husband. Meanwhile she meets Kamran, manager of a fast food, and her life gets on a new path. Kamran, is a seemingly handsome person, who knows the French language, which is a symbol of love. He knows romantic behaviors and gives calm and peace to Mina, but eventually betrays her. The monologues of Mina on the screen quickly give us signs of contact with an unconditional, weak, passive and dependent person. Kamran destroys the love in her heart by betraying Mina and abusing her fragile spiritual conditions and, most of all, her loneliness. Mina is a symbol for women who have not learned to be independent, and her happiness is subject to presence of a man in her life. Mina is the symbol of all women who do not know how to be happy, and their existence is ingrained in being with strong gender.

\subsection{Samples}

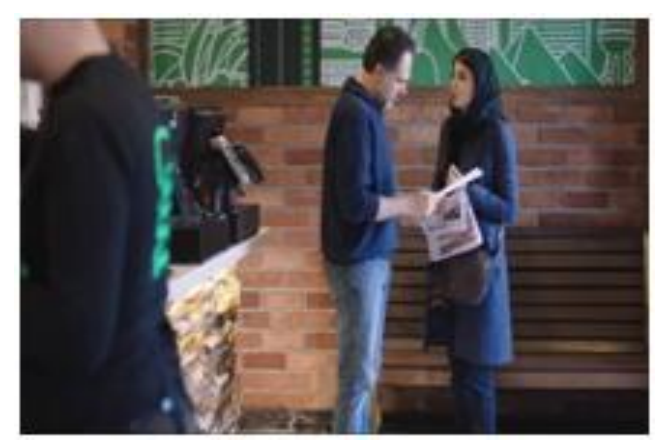

1: Mina fills out the employment form in the restaurant. He gives the reception letter to the fast food manager, Kamran Hassibi, and says: excuse me, I wrote bachelor's degree in the education part, but I left my studies when I was in the last term. In fact, there was a few remaining units until the bachelor's degree

Kamran: "Are you newly separated?"

Mina: Yes, but a little bit of its paperwork has not been completed yet.

Kamran: How many units exactly?

Mina falls silent and both smile.

Kamran: Okay. Do not worry about it.

Analysis:

Fast food was not a part of Iranian food culture in the past, and it is an imported culture that has many fans, which reflects the modernity of Iranian society. Looking for job by Mina and her half completed education reflects the lack of family support, and the inability of her family to pay for her expenses lack of social welfare and low cultural level of society. Therefore, a kind of causal relationship can be understood in this sequence, as the reason for failure to meet the financial and social needs of the 
family and the individual (here Mina) lies in the society, and the effect of this phenomenon is the search for enamel in fast food, therefore, in accordance to Peirce's theory, from the viewpoint of semiotics there is a kind of causal relationship between signifier and signified. Also, the appearance and costumes of Mina and her divorce indicates her non-traditional life, so another signifier and signified relationship can be imagined. Hence, in the present sequence, there is a kind of index relationship between the cause and the effects. In addition to this index relationship, within the sequence, due to the similarity of each picture with the object, the iconic relationship is also understandable.

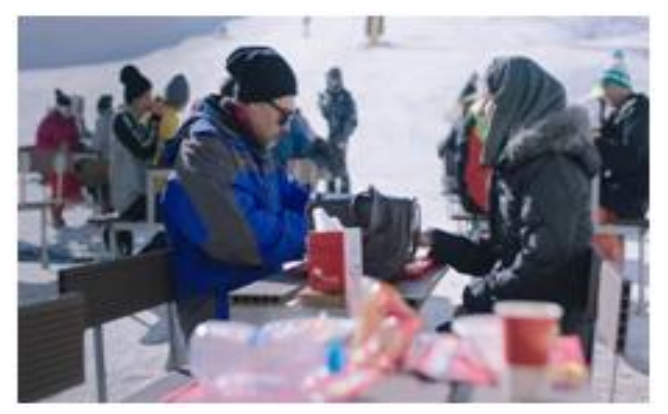

2: Mina sits on a chair in a ski resort, and Kamran comes in with a bowl of lentils in his hand and says some sentences in French.

When you went there, I wanted to give you a proper dine, what are these?

Mina: I had a craving for ice cream.

Mina: You speak French very well, lucky you, it has been my dream to be able to speak French like that. It's so beautiful; your accent also is so beautiful.

Kamran: Thank you

Mina: Do you like France.

Kamran: Not so much, but because I had to go to Quebec for study, I had to learn it.

Kamran: I'll teach you. But I have to say it now, I'm strict a bit. We start right now with first lesson.

Analysis:

This sequence shows that Iranian youth are trying to separate themselves from their traditional life and past culture and turn to modern (today) entertainments. Iranian men and women have chosen different apparel for this.

Selecting the ski resort and wearing ski suits, speaking in French, are the differences that make a person go toward modernity from tradition. In this sequence, from a semiotic point of view, there is a relationship proportional to Peirce's approach and the signifier and signified relation stands between the sign and the subject.



3: Mina and Kamran sit side by side and Kamran plays on the Tombak.

The narrator is Mina who talks to herself.

(Nights and days were passing with an unrealistic haste, we were on another world, I was the end dreaming, sleep end of the loneliness of the cradle -to find comfort - safety - dreams of love - luck happiness - do not wake me up.) 
Analysis:

In this sequence, costumes of Mina and Kamran are not traditional. Both of them wearing jeans and Kamran's T-shirt show their non-traditional attitude and life. The way in which they sit next to each other points to the fact that the relationship between men and women and their kind of interaction, has violated the traditions to a certain extent, and thus, according to Peirce's (1931) viewpoint, there is an index relationship between cause and effect in terms of semiotics. In addition to the index relationship, because of the similarity of each sequence with the intended object, the symbolic relationship is also understandable.

Also, using the traditional Tonbak instrument pictures a part of the traditional Iranian culture, which was a kind of man's recreation in the past, but nowadays, with the advancement of science and technology and culture, it is not only a matter that no longer knows gender and is not specifically for men, but is considered as a kind of value for a person.

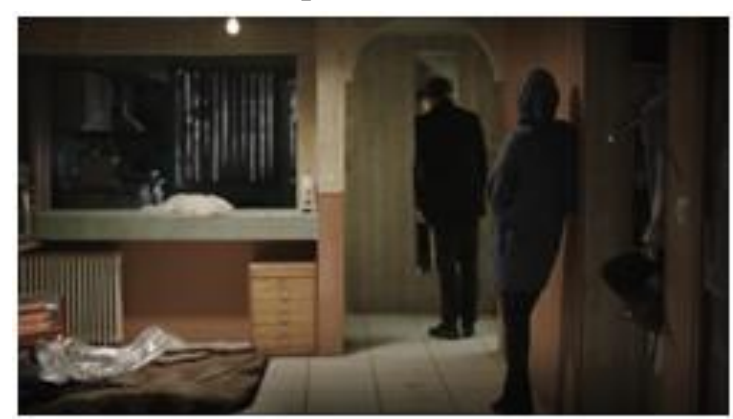

4: Kamran puts on his clothes in front of the mirror in the apartment.

Kamran: I'm not going with my car, my friend said he is going to come after me, I told him what are you talking about? I will come myself.

He Said no. First you fork out that piece of junk. I don't want to leave him standing for too long.

Do we have a comb?

Mina: Yeah, she takes out her hairbrush from bag and gives it to Kamran.

Just when I came, I parked the car in a hurry, gives switch to Mina, see what you can do.

Mina talks to herself: "How deserving and elegant, the most beautiful man I ever saw in my life, but it's a pity that he's not mine. He's not mine."

Analysis:

In this sequence, the relationship between Kamran and Mina reveals a kind of antagonism and confrontation with Iran's social and cultural traditions. In terms of semiotics, there is a sort of deviation from the norms, and connection is symbolic between the sign and the object, because their relationship is contrary to the usual social norms in society and is in contrast to the Iranian cultural and religious manners and therefore it is considered as a symbol of antagonism to social norms. This indicates that the social restrictions have not been accepted. Therefore, the symbolic relation between the sign and the meaning is observable.

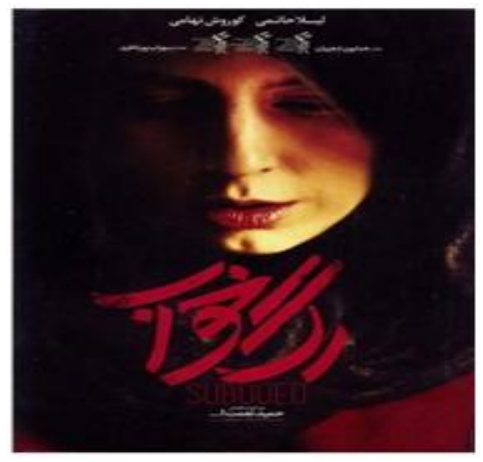

\section{Analysis:}

The picture of film's leading actress has been used as a way of encouraging audience to watch it. The dark background of this poster is painting a black picture of movie's story. Peirce points out, in his semiotic principles, that no sign can be completely iconic, index, or symbolic, and in fact, any sign 
can be a combination of all three, which in each case one of them dominates the other two. The main background is chosen to be a black design, which is completely in accordance with the story of the film. The black symbol in the framework of the semiotic principles of Peirce is well matched with its meaning, tyranny and sacrifice, so another symbolic link can be seen in this image.

In most of cultures, red color is the symbol of love. Using the red color for the movie's title reflects the main emphasis on the movie's title and can also be a factor in attracting audiences to watch the movie.

In Peirce's semiotics, even thoughts are signs. Therefore, the design of the three Crystal Simorghs of the Fajr Film Festival in the image illustrates that film has won in three artistic categories. Mentioning the name of the director and the singer can also attract the audience. Also, using the white color to show the above points suggests relaxing and reassuring the viewer to watch the movie.

Table1: Different sign frequency in Rag-e khab

\begin{tabular}{|l|l|l|}
\hline Row & \multicolumn{1}{|c|}{ Sing types } & Frequency \\
\hline 1 & Iconic signs & 63 \\
\hline 2 & Index signs & 57 \\
\hline 3 & Symbolic Signs & 15 \\
\hline
\end{tabular}

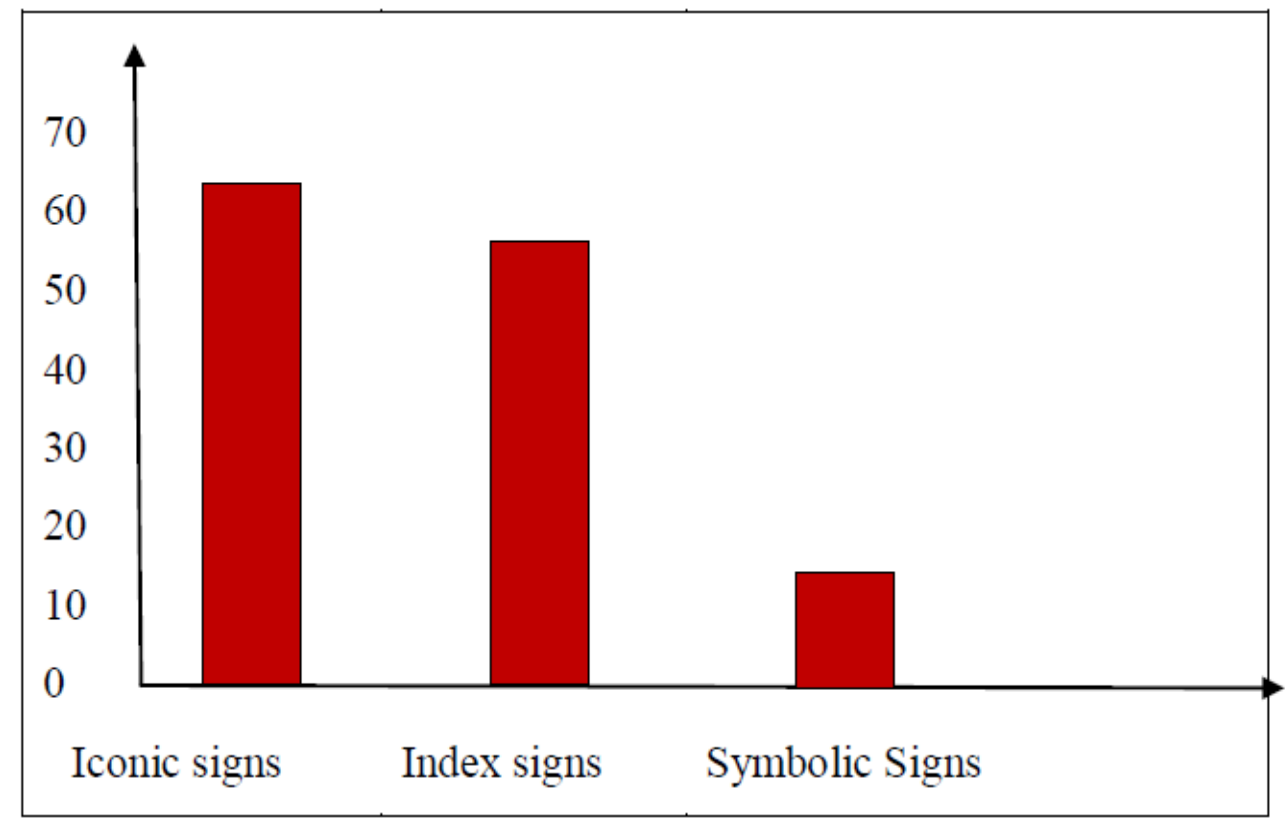

Figure1: The frequency distribution of different kinds of sign in Rag-e khab

\section{CONCLUSION}

The present study examined the semiotics of the movie Rag-e Khab from the viewpoint of Peirce. It is found that the director of the film, like other social film directors working today, has produced the film in order to examine and illustrate the existing challenges, focusing on a social problem. From the film sequences, it became clear that the social and cultural issues of today and the way youths communicate are being examined and criticized. In this research, the cause and effect relationship, which is the same index communication, has been used to convey meaning and concepts to the audience, because the director has produced the film for the specific purpose of showing the social problems of today's women. Thus, through the film and the image, he has tried to demonstrate a problem and the reason for the emergence of the problem is also related to an effect in the environment and society, therefore, most of the relationships between the signifier and the signified are mostly index type, and given that the analyzed data in this research are image and sequences, all sequences have a symbolic relationship with the object and their relationship with object is based on similarity. The index signs are used extensively in this film, and symbolic signs are also seen in some cases. In the table, the numbers of each sign are listed separately. This table shows that usage of the iconic signs is statistically the highest, and then the index signs and then the symbolic signs have had the least usage. The results also indicate that the representation of gender in this film is female- 
oriented. All the sequences focused on today's women's issues, their personality and their attitudes, the way they communicate with men, is evident in all the sequences.

\section{REFERENCES}

[1] Barth, R. (1964). Image- music text. (6 ${ }^{\text {th }}$ ed.), London: London University Press.

[2] Barth, R. (1991), Elements of Semiotics, translated by Majid Majidi, Tehran: Alhoda.

[3] Duboa, N. (1983), Le Processus interprtatif. Introduction a la semiotique de Ch. S. Peirce Mardaga.

[4] Kazemi, F. and Ghasemi Ateni, N. (2016), "A Semio- Semantic Study fo Reute's (New Agency) Photographs", International Journal of Advanced Research, Vol. 4, No. 6, PP. $73-77$.

[5] Peirce, C. S. (1906), "the basis of pragmaticism, collected papers", Vol. 1-6, Cambridge) 1031 (S. Peirce, $\mathrm{C}$ (Massachusetqs): Harvard University press.

[6] Saussure, F. (2001), Fundamentals of Constructivism in Linguistics, translated by Kourosh Safavi, Tehran: Soureh Mehr Publication.

[7] Saussur, F. (1916). Course in General Linguistics. ( $8^{\text {th }}$ ed.), London: London University Press.

[8] Sebeok, A. T. (2001), Signs: An Introduction to semiotics, (second ed.), Torento: University of Torento Press.

[9] Sharam, p. and Gupta, p. (2015), "Semiotic analysis of Indian television. Advertisements and its impact on consumers: an exploratory study", Essachess, Journal for communication studies, vol. 8, 1(15),pp. 71-90.

[10] Sojoodi, F. (2008), Applied Semiotics, ( $2^{\text {nd }}$ ed.), Tehran, Nazareh elm publications.

[11] Zamiran, M. (2003), An Introduction to the Semiotics of Art, (1 ${ }^{\text {st }}$ ed.), Tehran, Ghesseh publications.

\section{AUTHORS' BIOGRAPHY}

Foroogh Kazemi was born in Iran in 1974. She is head of linguistics department and associate professor of linguistics in Central Tehran Branch, Islamic Azad University in Iran. She has published 54 articles (most of them cited in google scholar) and 15 books in linguistics. She has been teaching at graduate and Ph.D. levels in linguistics. Dr. Kazemi has been advisor, reader and referee of many linguistic theses and judge of articles in journal of linguistics. She is a member of research council in faculty of foreign languages at Central Tehran Branch. She is also a member of editorial board, quarterly of language and literature: Parneyan Kheyal. Her areas of interest are functional linguistics, sociolinguistics, typology and discourse analysis.

Shohreh Dalaee was born in Iran. She is MA of linguistics, at Central Tehran Branch, Islamic Azad University. She has written a book about discourse analysis and has published an article about gender discourse in 2017.

Citation: Dr. Foroogh kazemi, Shohreh Dalaee. The Semiotic Study of Rag-E Khab from the Peirce's Perspective. "International Journal on Studies in English Language and Literature (IJSELL), vol 7, no. 9, 2019, pp. 1-7 doi: http://dx.doi.org/10.20431/2347-3134.0709001.

Copyright: (C) 2019 Authors. This is an open-access article distributed under the terms of the Creative Commons Attribution License, which permits unrestricted use, distribution, and reproduction in any medium, provided the original author and source are credited. 